\title{
Tuberculous pericarditis : experience in a community hospital
}

\author{
Fernando Peixoto Ferraz de Campos ${ }^{a}$, Aloísio Felipe-Silvab ${ }^{b}$ Leonardo Gomes Fonsecac, \\ Luiz Fernando Seguro $^{d}$, Antônio Fernando Barros de Azevedo Filhoc
}

Campos FPF, Felipe-Silva A, Fonseca LG, Seguro LF, Azevedo Filho AFB. Tuberculous pericarditis: experience in a community hospital. Autopsy Case Rep [Internet]. 2011;1(1):3-12.

\section{ABSTRACT}

Tuberculosis is a major public health problem worldwide. In Butantan district of São Paulo city, the average incidence over the last 7 years was 47.7 new cases $/ 100,000$ inhabitants. Tuberculous pericarditis is a serious form of extrapulmonary tuberculosis in which diagnosis is often difficult. Diagnosis needs to be reached fast and accurately once tuberculous pericarditis has high morbimortality rates if untreated. We report the experience of a community hospital on tuberculous pericarditis with emphasis on the clinical presentation and diagnostic procedures. From 2003 to 2010, 59 patients were diagnosed with pericarditis, 6 $(10.16 \%)$ of which had tuberculous pericarditis. Demographic, clinical, imaging, laboratorial, microbiological and histological data were reviewed. There were 4 female $(66.7 \%)$ and 2 male $(33.3 \%)$ patients. Age ranged from 17 to 62 years (median= 25.5). One patient $(17 \%)$ had HIV co-infection. Five patients $(83.3 \%)$ had cardiac tamponade on Echocardiogram. Histopathology was confirmatory in 4 cases $(66.7 \%)$ while cultures were positive in 2 cases $(33.3 \%)$. Four patients $(66.7 \%)$ had definite and $2(33.3 \%)$ had probable diagnosis of tuberculous pericarditis. One patient $(17 \%)$ died during admission. Reuter's diagnostic index was $\geq 6$ in 5 patients $(83.3 \%)$. We concluded that the clinical picture, Reuter's diagnostic index, signs of cardiac tamponade on Echocardiogram and the pericardium biopsy were the most important features for the diagnosis of tuberculous pericarditis.

Keywords: Tuberculosis; Pericarditis; Pericarditis, tuberculous; Diagnosis.

\footnotetext{
a Department of Internal Medicine of the Hospital Universitário - Universidade de São Paulo, São Paulo/SP, Brazil.

b Service of Anatomic Pathology of the Hospital Universitário - Universidade de São Paulo, São Paulo/SP, Brazil.

c Department of Internal Medicine of the Hospital das Clínicas - Faculdade de Medicina, Universidade de São Paulo, São Paulo/ SP, Brazil.

d Instituto do Coração - Hospital das Clínicas - Faculdade de Medicina Universidade de São Paulo, São Paulo/SP, Brazil.
} 


\section{INTRODUCTION}

Tuberculosis is a major public health problem worldwide. It is estimated that about 4 to 10 million new cases occur per year, with 1 million deaths. ${ }^{1}$ According to the World Health Organization, the incidence of the disease is stable in developed countries, after a period of growth observed in the 90's. However, the global incidence increases around 1\% a year, especially because of its growth in regions of poor socioeconomic conditions, where co-infection by the HIV confers high rates of morbimortality to the disease. ${ }^{2}$

The incidence in Brazil between the years of 1990-2001 ranged from 75,000-85,000 new cases/year, falling to 60,000 new cases in 2002 , which represents an incidence coefficient of 50 to 99/100,000 inhabitants. ${ }^{3}$ Some countries in Africa present a coefficient of 300 or more and in the USA and Canada this coefficient is 10 . In the state of São Paulo, 15,344 new cases were recorded in 2009, and the annual average between 2004 and 2009 was 16,121 new cases per year. In São Paulo city 5,777 new cases of tuberculosis were recorded in 2009, and the average of the previous 5 years was 6,140 new cases per year. In the district of Butantan, where the Hospital Universitário da Universidade de São Paulo (HU-USP) is located, 1,274 new cases of tuberculosis were recorded from 2003 to 2010 , with an average incidence coefficient of $47.7 / 100,000$ inhabitants $^{\mathrm{e}}$.

The current epidemiologic scenario of the disease, considering the advance of HIV infection and other forms of immunosuppression, and the increasing incidence in populations with poor health conditions worldwide, stresses the importance of extra pulmonary forms of tuberculosis, among which the Tuberculous Pericarditis.

Tuberculous pericarditis is a rare form of extra pulmonary tuberculosis. Data from the 60's showed tuberculous pericarditis in 0.4 to $1.1 \%$ of all autopsies, and in 7.3 to $11 \%$ of the cases with acute pericarditis. ${ }^{4}$ In developing countries, tuberculosis is the main cause of infectious pericarditis, and an incidence of 70 to $80 \%$ of the infectious pericarditis is reported in Africa, $90 \%$ in HIV positive patients. ${ }^{5}$ In a series in South Africa, tuberculosis was the etiology found in $69.5 \%$ of the 233 cases submitted to pericardial effusion drainage ${ }^{6}$ Among the causes of pericarditis in developed countries, tuberculosis accounts for about 4 to $5 \% .^{5,7}$
The tuberculous pericarditis is classified as definite or probable according to one or more of the following criteria:

Definite when there is: (1) isolation of $M y$ cobacterium tuberculosis of the pericardial effusion or fragment of biopsy (presence of Acid-fast bacilli $(A F B)$ in the Ziehl-Neelsen staining or positive culture in the fragment); or (2) demonstration of granulomas in the histological exam of the pericardial biopsy.

Probable when there is: (1) evidence of pericarditis in patients with tuberculosis demonstrated in any other site; (2) pericardial effusion characterized by exudate with high Adenosine Deaminase (ADA) activity and/or (3) good response to antituberculous chemotherapy, in the absence of any other obvious cause for the pericardial effusion. ${ }^{8}$ Tuberculous pericarditis is a form of extra pulmonary tuberculosis of considerable gravity, with a mortality rate of $85 \%$ in 6 months if untreated ${ }^{9}$, and of $3 \%$ to $40 \%$ when treated. ${ }^{10}$ Therefore, the diagnosis should be accurate and fast. However, this is often difficult due to the low sensitivity in detecting mycobacterium in samples, slowness in growing mycobacterium in cultures and to the need for invasive techniques, which often require specialized personnel and centers.

\section{PATIENTS AND METHODS}

Data from patients discharged between January 2003 and December 2010 with diagnosis of pericarditis and/or tuberculosis were recovered from hospital epidemiological information service and from the records of the Internal Medicine infirmary. Fifty nine patients with pericarditis and 87 patients with tuberculosis were identified. Six patients had tuberculous pericarditis $(10.16 \%$ of pericarditis cases and $6.9 \%$ of tuberculosis cases). From these six cases, $4(66.7 \%)$ had definite diagnosis of tuberculous pericarditis and $2(33.3 \%)$ had probable tuberculous pericarditis.

The following data were retrieved from medical charts: demographic data, clinical history, length of symptoms from onset to hospitalization, data from physical examination, imaging exams, ADA activity of the pericardial and pleural effusion (when present), mycobacterium cultures from the pericardial effusion or pericardial tissue, and histopathology of the pericardium.

\footnotetext{
e Data supplied by Unidade de Vigilância à Saúde - Butantan, da Secretaria de Higiene e Saúde da Prefeitura Municipal de São Paulo, SP. 
Pericardial biopsies were performed through subxiphoid approach. Histopathological slides from all cases submitted to biopsy were reviewed by a second pathologist. All histopathological slides were stained with Hematoxilin-Eosin and Ziehl-Neelsen for AFB research.

\section{RESULTS}

Laboratorial and some demographic data of the studied cases are summarized in Table 1. Age ranged from 17 to 62 years. The average was 30 and the median was 25.5 years. Four $(66.7 \%)$ patients were female and $2(33.3 \%)$ were male.

Mortality during admission was $17 \%$. One patient (case 2) died due to complications related to tuberculous pericarditis. Autopsy confirmed tuberculosis in the pericardium (Figures 1 to 3 ), lung, pulmonary hilar lymph node and foci of hematogenic dissemination in the lungs and both adrenals with positive AFB in all these sites. This patient also had hepatosplenic schistosomiasis. The other patients were released and followed up in the outpatient clinic.

Reported length of symptoms ranged from 6 to 16 weeks, with an average of 9.5 weeks. Prevalence of the reported symptoms and physical examination findings are presented in Table 2. A comparison of the frequency of findings in this study with data from Reuter's study ${ }^{6}$ is shown in Table 3.

Table 1 - Clinical and laboratorial presentation

\begin{tabular}{|c|c|c|c|c|c|c|}
\hline & Case 1 & Case 2 & Case 3 & Case 4 & Case 5 & Case 6 \\
\hline Gender & $\mathrm{F}$ & $\mathrm{M}$ & M & $\mathrm{F}$ & $\mathrm{F}$ & $\mathrm{F}$ \\
\hline Age (years) & 17 & 62 & 27 & 27 & 23 & 24 \\
\hline $\begin{array}{l}\text { Length of symptoms } \\
\text { (days) }\end{array}$ & 45 & 120 & 21 & 120 & 60 & 60 \\
\hline Fever & + & + & + & + & + & + \\
\hline Weight loss & + & + & - & + & + & + \\
\hline Night sweats & - & - & + & - & + & - \\
\hline Coughing & - & - & - & + & + & + \\
\hline Dyspnea & - & - & - & + & - & + \\
\hline Thoracic pain & - & - & + & + & - & + \\
\hline Jugular stasis & + & + & + & + & - & - \\
\hline $\begin{array}{l}\text { Tachycardia/hypotension/ } \\
\text { tachypnea }\end{array}$ & + & + & + & + & + & + \\
\hline $\begin{array}{l}\text { Hypophonetic heart } \\
\text { sounds }\end{array}$ & + & + & - & + & - & - \\
\hline Chest X-ray & + & $\begin{array}{l}\text { Bilateral pleu- } \\
\text { ral effusion }\end{array}$ & $\begin{array}{l}\text { +/pleural effu- } \\
\text { sion }\end{array}$ & $\begin{array}{c}\text { +/effusion } \\
\text { Pleural } \\
\text { bilateral }\end{array}$ & $\begin{array}{l}+/ \text { bilateral } \\
\text { interstitial pro- } \\
\text { cess }\end{array}$ & $\begin{array}{l}\text { Bilateral } \\
\text { pleural } \\
\text { effusion }\end{array}$ \\
\hline Alt ECG & + & + & - & + & + & - \\
\hline Echo with tamponade & + & + & + & + & + & $\begin{array}{l}\text { Pericar- } \\
\text { dial thick- } \\
\text { ening }\end{array}$ \\
\hline Pericardial effusion & Exudate & $\begin{array}{c}\text { Exudate /PMN } \\
\text { Serosanguine- } \\
\text { ous }\end{array}$ & $\begin{array}{l}\text { Exudate Lym- } \\
\text { phomonuclear }\end{array}$ & $\begin{array}{l}\text { Exudate } \\
\text { PMN }\end{array}$ & - & - \\
\hline
\end{tabular}


Table 1 - Clinical and laboratorial presentation

\begin{tabular}{|c|c|c|c|c|c|c|}
\hline $\begin{array}{l}\text { Pericardial effusion ADA } \\
(\mathrm{U} / \mathrm{I})\end{array}$ & 6 & 30 & 73 & 43 & NA & - \\
\hline Pleural effusion ADA (U/I) & - & 8 & 60 & - & - & 140 \\
\hline AFB culture & - & - & - & $\begin{array}{c}+ \text { (pericar- } \\
\text { dium tissue })\end{array}$ & $+(\mathrm{BAI})$ & - \\
\hline \multirow[t]{2}{*}{ Pericardial biopsy } & $\begin{array}{l}\text { CGP with } \\
\text { CN }\end{array}$ & $\begin{array}{c}\text { CGP with CN } \\
\text { AFB - }\end{array}$ & NSCP & $\begin{array}{l}\text { CGP with } \\
\text { CN AFB- }\end{array}$ & CGP without & - \\
\hline & AFB- & & & & $\begin{array}{l}\text { CN } \\
\text { AFB- }\end{array}$ & \\
\hline HIV & negative & negative & positive & negative & negative & negative \\
\hline Diagnosis & definite & definite & probable & definite & definite & probable \\
\hline Reuter's DI & 6 & 6 & 9 & 9 & 10 & NA \\
\hline
\end{tabular}

Tachycardia= resting heart rate $>100 \mathrm{bpm}$, hypotension= PAS $\leq 100 \mathrm{mmHg}$, tachypnea $=\mathrm{FR}>16 \mathrm{rmpm}$ Chest X-ray $(+)=$ increase in heart image

ECG $(+)=$ complex QRS of low voltage, alteration in the T wave and the ST segment, sinus tachycardia $\mathrm{CGP}=$ Chronic Granulomatous Pericarditis; $\mathrm{CN}=$ caseous necrosis; NSCP= nonspecific chronic Pericarditis.

$\mathrm{BAl}=$ bronco alveolar lavage.

$\mathrm{ID}=$ diagnostic index proposed by Reuter.

NA $=$ Not available

Table 2 - Prevalence of clinical findings (6 cases)

\begin{tabular}{lll}
\hline & Characteristic & Prevalence (\%) \\
\hline Symptoms & Fever & 100 \\
& Weight loss & 83 \\
& Sweating & 60 \\
& Thoracic pain & 50 \\
Dry cough & 50 \\
Digns & Dyspnea & 17 \\
& Syncope & 17 \\
Echocardiographic signs of tamponade & 83 \\
Tachycardia & 67 \\
Pleural effusion & 67 \\
Jugular stasis & 67 \\
Hypophonesis of cardiac sounds & 50 \\
\hline & Hypotension & 33 \\
\hline
\end{tabular}


Table 3 - Comparison of the frequency of findings in our study with data from the study with 162 cases of tuberculous pericarditis

\begin{tabular}{lcc}
\hline Characteristic & $\begin{array}{c}\text { Reuter et al. (1995-2001) } \\
(\%)\end{array}$ & HU-USP (2003-2010) \\
\hline Fever & 75 & 100 \\
Weight loss & 79 & 83 \\
Dyspnea & 76 & 60 \\
Thoracic pain & 27 & 50 \\
Coughing & 90 & 50 \\
Sweating & 72 & 17 \\
Syncope & Not reported & 17 \\
Tachycardia & 74 & 83 \\
Hypophonesis of cardiac sounds & 75 & 67 \\
Hypotension & 6 & 67 \\
Jugular stasis & 78 & 67 \\
Pleural effusion & 38 & 50 \\
Tamponade & 90 & 33 \\
\hline
\end{tabular}

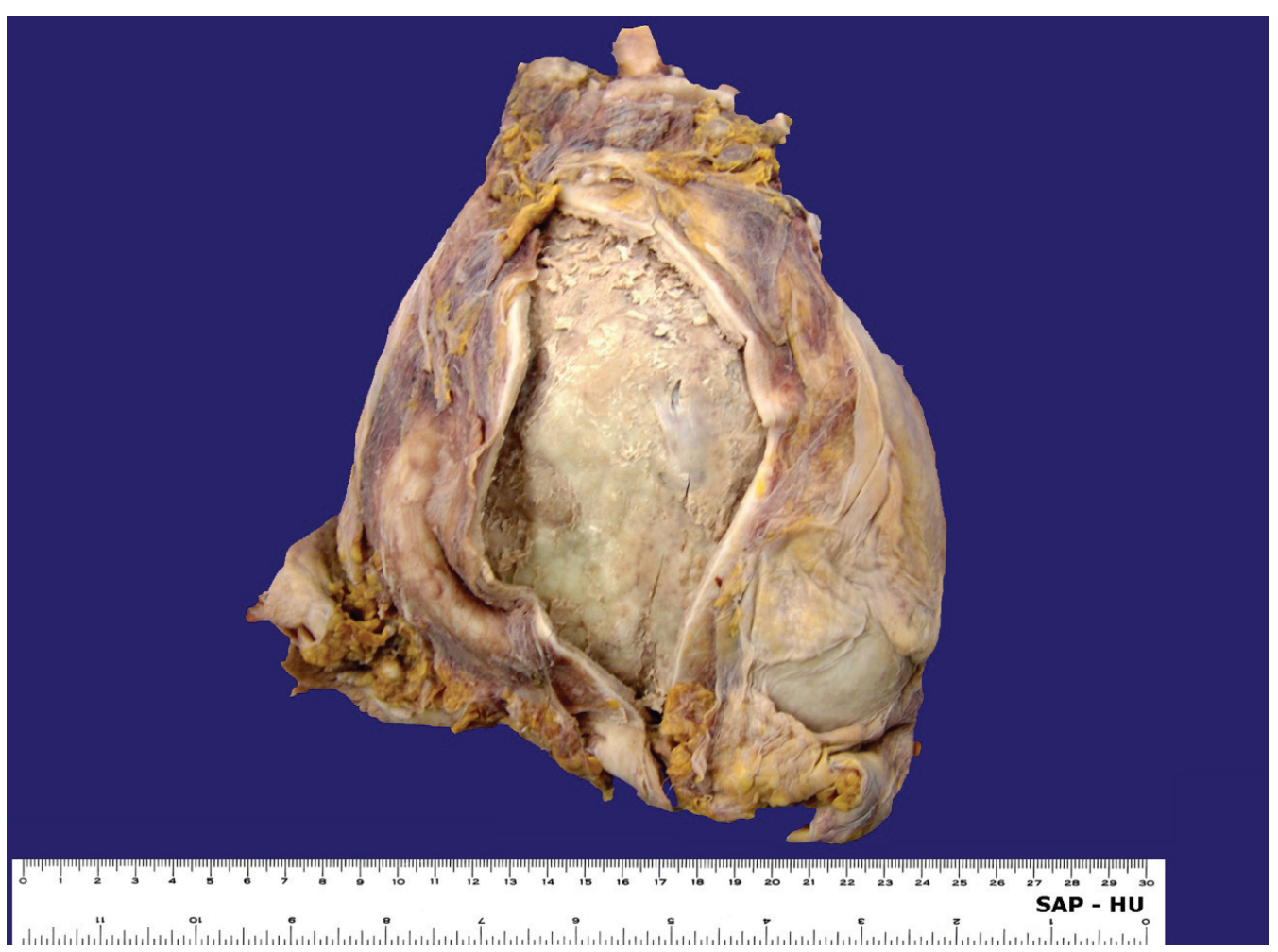

Figura 1 - Anterior view of heart and thickened parietal pericardium 


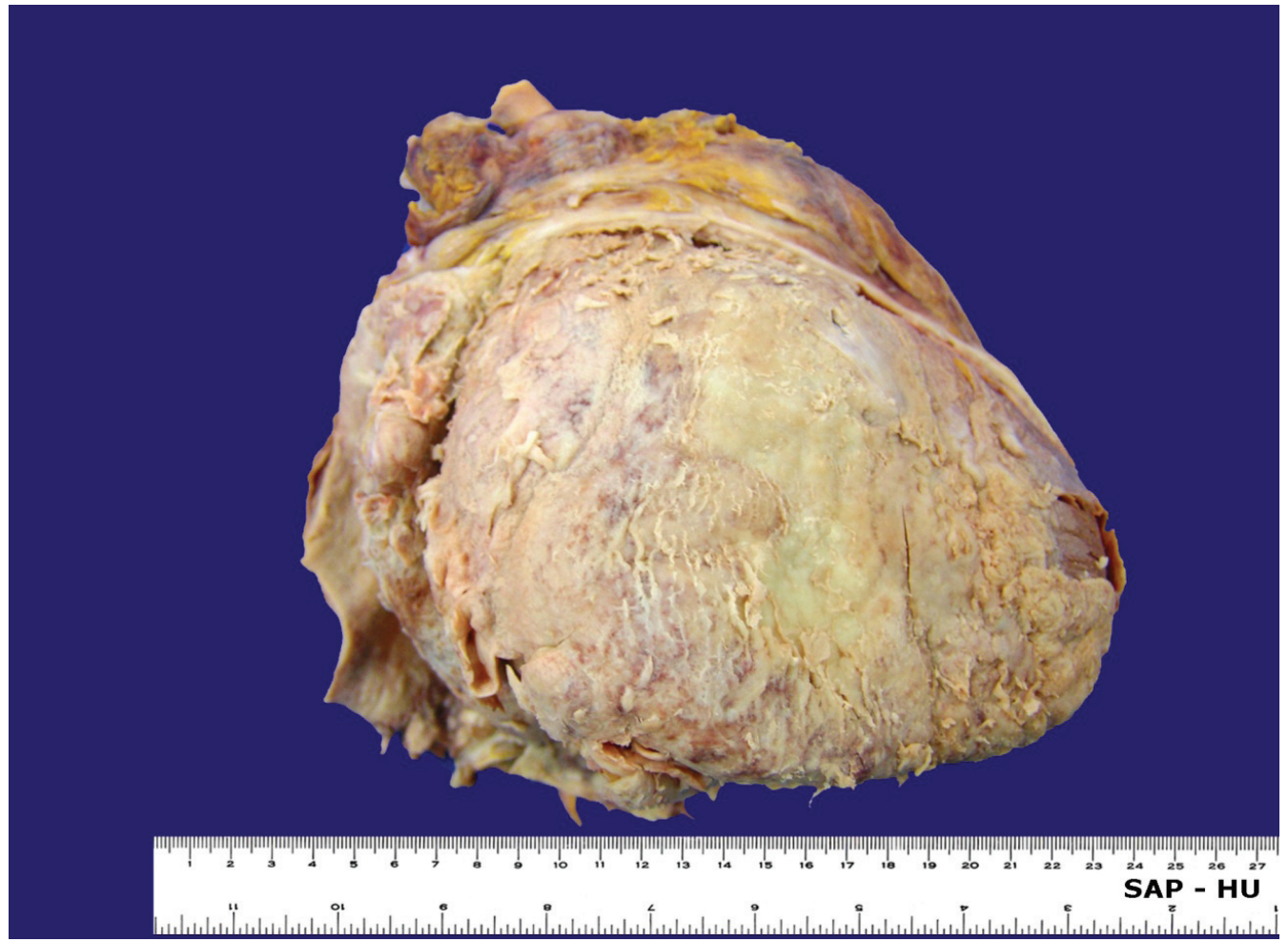

Figure 2 - Open view of pericardial sac showing thickening of visceral pericardium by fibrin

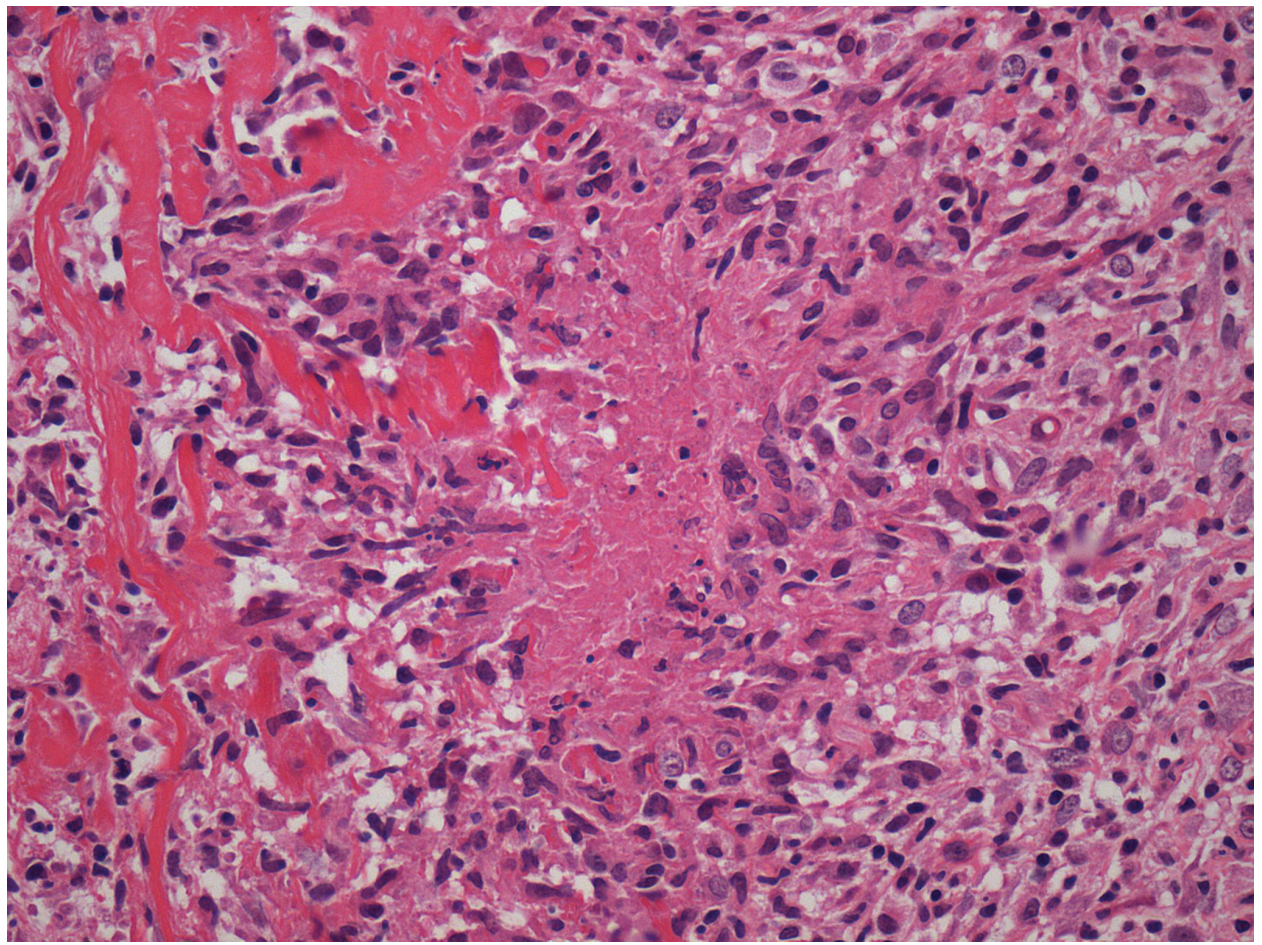

Figure 3 - Necrotizing granuloma within pericardial tissue (HE, 400x)

In 4 out of 5 cases submitted to biopsy ( $80 \%$ of the patients) the histopathological exam was confirmatory (cases 1, 2, 4 and 5). In case number 3 , the diagnostic conclusion was defined through the ADA activity and confirmed tuberculosis in another site (lung); in case number 6 , there was evidence of tuberculosis in another site (pleura), tamponade pericardial effusion and clinical improvement after specific treatment. Overall, tuberculous pericarditis was observed concomitantly with tuberculosis in other sites in $67 \%$ of the cases (cases 2, 3, 5, and 6). 


\section{DISCUSSION}

Pericardial involvement in tuberculosis occurs through dissemination of Mycobacterium tuberculosis from mediastinal, peribronchial and paratracheal lymph nodes or through hematogenic spread from primary tuberculosis infection. The infection rarely affects the pericardium by contiguity of pulmonary lesion or through hematogenic spread from distant lesions like bones or genitourinary tract. The immune response to viable bacilli that penetrate the pericardium is responsible for morbidity. Four pathological stages of tuberculous pericarditis are recognized: (1) Pericarditis with fibrinous exudation and polymorphonuclear leukocytes, relatively abundant mycobacteria, early granuloma formation with loose organization of $\mathrm{T}$ lymphocytes and macrophages; (2) serosanguineous effusion with a predominantly lymphocytic exudate with monocytes and foam cells; (3) absorption of the pericardial effusion, organization of granulomatous caseation and pericardial thickening caused by fibrin, collagen and ultimately fibrosis; (4) constrictive scarring fibrosis of the visceral and parietal pericardium encasing the heart in a fibrocalcific mold that hinders diastolic filling leading to the constrictive pericarditis syndrome. $^{12}$

When facing the clinical presentation of large pericardial effusion, establishing the etiological diagnosis becomes urgent. In case of tuberculous etiology, immediate specific treatment dramatically improves prognosis. Since this diagnosis is often difficult, the physician is forced to start therapeutics based on clinical data despite the toxicity and the long course of the specific treatment. ${ }^{13}$

The development of tuberculous pericarditis is frequently insidious and followed by nonspecific systemic symptoms such as fever, night sweats, fatigue, weight loss, thoracic pain, coughing and dyspnea ${ }^{14,15}$. In more advanced stages, signs and symptoms compatible with pericardial tamponade or constrictive pericarditis may appear. An antecedent of previous tuberculosis may be referred in the clinical history.

Constitutional symptoms such as fever and weight loss, as well as respiratory symptoms such as coughing, dyspnea and thoracic pain characterize the clinical presentation of tuberculous pericarditis. The lack of specificity of these findings demonstrates how difficult this diagnosis is. We observed that some physical exammination findings may be useful when suspecting of tuberculous pericarditis: tachycardia (in 67\%), hypophonesis of cardiac sounds (in 50\%), jugular stasis (in $67 \%$ ) and hypotension (in $33 \%$ ). In a South African study with 162 patients with tuberculous pericarditis, the same findings were found in $54 \%, 55 \%, 6 \%$ and $78 \%$, respectively. ${ }^{6}$ (Table 3 )

We found that $83 \%$ of our patients presented with evidence of cardiac tamponade. These is consistent with a rate of up to $90 \%$ reported by others and demonstrates the difficulty in making the diagnosis of tuberculous pericarditis prior to the appearance of hemodynamic complications ${ }^{6}$.

In Reuter et al series of 162 cases of tuberculous pericarditis, laboratorial significance was found in high serum protein and serum globulin (globulin $>4 \mathrm{~g} / \mathrm{l}$ results in OR of 15.1 - these values tend to be higher in HIV positive patients). Peripheral blood total leukocyte counts, neutrophil and lymphocyte numbers were significantly lower in patients diagnosed with tuberculous pericarditis ${ }^{6}$. No patient presented leukocytosis in the blood-cell count in that study.

Reuter et $\mathrm{al}^{6}$, in a study on pericardial effusion (110 patients with definite diagnosis of tuberculous pericarditis and 54 with diagnosis of nontuberculous etiology), suggest a diagnostic index (DI) with 5 variables observed at patient's admission. This index is composed of weight loss $(D I=1)$, night sweats ( $D I=1)$, fever $(D I=2)$, serum globulin $>4 \mathrm{~g} / \mathrm{dl}(\mathrm{DI}=3)$ and leukometry (on peripheral blood) $<10000 / \mathrm{mm} 3(\mathrm{DI}=3)$. The sum of these scores results in a diagnostic index. DI $\geq 6$ results in diagnosis of tuberculous pericarditis with $86 \%$ sensitivity and $84 \%$ specificity.

Chest X-ray may show an enlarged heart image in up to $90 \%$ of the cases, depending on disease stage. Calcium deposits around the image of the heart may be seen in cases of constrictive pericarditis. ${ }^{16}$ Active pulmonary tuberculosis may be present in $30 \%$ of the cases and pleural effusion in $38 \%-60 \%{ }^{6,8}$. We found pleural effusion in $67 \%$ of the patients.

Electrocardiogram (ECG) is abnormal in almost all cases of pericardial effusion, usually with nonspecific ST-T wave changes. The elevation of the ST segment, which characterizes acute pericarditis, only appears in $9-11 \%$ of the cases. The presence of microvoltage (voltage $<5 \mathrm{~mm}$ in limb leads and $<10 \mathrm{~mm}$ in precordial leads) suggests the presence of large pericardial effusion. Cardiac tamponade is unlikely in the absence of ECG microvoltage. ${ }^{17}$ Atrial fibrillation occurs in $4 \%$ of the 
cases and electrical alternans that occurs in cardiac tamponade is uncommon ${ }^{8}$. In our sample, the ECG was altered in $67 \%$ of the cases. The presence of low voltage complexes, alterations in the $T$ wave and segment ST and sinus tachycardia were the most frequent findings.

The most characteristic echocardiographic data of tuberculous pericarditis when compared to other causes of pericarditis were pericardial thickening $>5 \mathrm{~mm}$, fibrinous debris in the pericardial space and cardiac tamponade. ${ }^{6}$ Signs of cardiac tamponade was the most frequent finding at echocardiography in our series ( $83 \%$ of the patients).

According to Light's criteria ${ }^{18,19}$ the pericardial effusion of tuberculous pericarditis is an exudate which, in $80 \%$ of the times is slightly hemorrhagic $^{8}$, with cellularity depicting lymphocytic predominance, despite descriptions of polymorphonuclear predominance ${ }^{20}$ and even cases of purulent tuberculous pericarditis ${ }^{6,21}$. A ratio between lymphocytes and neutrophils of the pericardial effusion $\geq 1$ has specificity, sensitivity, positive predictive value (VPP) and negative predictive value (VPN) of $79 \%, 73 \%, 86 \%$ and $61 \%$ respectively 6 , 21. We bear in mind that in the initial stage of the disease the cellularity of the pericardial effusion tends to be mainly polymorphonuclear.

The rate of detection of AFB in the pericardial effusion by direct fluid smear examination ${ }^{22}$ ranges from zero to $42 \%$. Culture of Mycobacterium tuberculosis by conventional routine is positive in $53 \%$, but rises to $75 \%$ when the pericardial effusion is inoculated into double-strength Kirchner culture medium at the bedside. ${ }^{23}$ Previous studies suggested that tuberculous pericarditis was more frequently diagnosed through pericardial biopsy when compared to analysis of the pericardial fluid. However, a prospective study in an endemic area showed that culture of the pericardial effusion confirmed tuberculosis more frequently than histology. ${ }^{24}$ It is observed that the probability of having a bacteriologic diagnostic is higher when analysis of the pericardial fluid and biopsy are performed in the initial stages of the disease ${ }^{24,25}$. Our data showed that sensitivity of microbiological analysis either by AFB direct staining or by culture was low: $0 \%$ and $33 \%$ respectively.

Pericardium biopsy was confirmatory in 4 out of 5 cases (case 6 was not submitted to biopsy), with $80 \%$ sensitivity since the biopsy showed nonspecific inflammatory findings in the only HIV positive patient.
Sensitivity of the histological exam for diagnosis of tuberculosis in pericardial fragments ranges from $10 \%$ to $64 \%$. In a Brazilian study, Vianna et al ${ }^{26}$ found histopathological results compatible with tuberculosis in $50 \%$ of cases in a series of 8 patients. The higher percentages are observed when the finding of granulomatous pericarditis with or without necrosis and/or presence of AFB is considered positive for tuberculosis ${ }^{6,8}$. In a prospective study of 36 patients with large pericardial effusion submitted to pericardium biopsy and microbiological study of the pericardial effusion, 25 patients presented diagnosis of tuberculosis. The histological patterns found in these 25 patients were: (1) granulomatous pericarditis with caseous necrosis AFB positive and AFB negative; (2) granulomatous pericarditis without necrosis AFB negative; (3) purulent pericarditis; (4) fibrotic pericarditis; (5) fibrinous pericarditis and (6) serous pericarditis. Granulomas with necrosis and AFB positive Ziehl-Neelsen staining were detected respectively in $48 \%$ and $30 \%$ of the biopsies. The culture for mycobacteria was positive in $50 \%$ of these cases ${ }^{6}$. Therefore, the association of cultures and histological analysis leads to a sensitivity of $83 \%$ for the diagnosis of tuberculous pericarditis. ${ }^{27,28}$ In our series the two patients who presented positive cultures also presented diagnosis of tuberculous pericarditis in the histopathological analysis.

The presence of HIV co-infection does not alter only the formation of granulomas but also the presence of necrosis. In HIV positive patients with low CD4 count $(<50$ cels $/ \mu l)$ unusual histological patterns for diagnosis of tuberculous pericarditis are observed such as purulent and serous pericarditis. The depletion of CD4 cells results in altered immune response with decreased granuloma formation. ${ }^{21}$ The only HIV positive patient in our sample (case number 3) presented a nonspecific chronic active pericarditis in the histopathological examination, not characteristic of tuberculous pericarditis. His diagnosis was established through the high ADA activity in the pericardial effusion and positive therapeutic response to the specific antituberculous therapy. However other cases have been reported regarding HIV positive patients with diagnosis of pericardial effusion in which biopsies were diagnostic for tuberculous pericarditis, with granulomas with caseous necrosis and presence of Langhans cells. ${ }^{29}$

Protein antigens of Mycobacterium tuberculosis induce a delayed hypersensitivity response. Local monocytes are essential to process and present bacilli antigens for $T$ lymphocytes, that, when stimulated, liberate lymphocynes. Among them 
there is the gama interferon (INFy), which activates macrographages influencing granuloma formation. The concentration of INFY in the pericardial effusion (when the cut-off considered is $50 \mathrm{pg} / \mathrm{ml}$ ) showed $92 \%$ sensibility and $100 \%$ specificity for tuberculosis. Using the cut-off of $200 \mathrm{pg} / \mathrm{ml}$ the sensitivity and specificity for the method was $100 \% .^{30}$ The concentration of INFY is not altered by the HIV coinfection. ${ }^{15,21,30}$

Adenosine deaminase (ADA) is the enzyme that catalyses the deamination of adenosine and desoxyadenosine into their respective inosine nucleosides. This reaction is the first step of a series of reactions responsible for the proliferation and differentiation of lymphocytes. The ADA activity indirectly reflects the population of $T$ lymphocytes involved in the inflammatory response and increases during mitogenic activity and antigen response. This enzyme is present in several animal cells, found from simple invertebrates to human beings, its concentration is 10 to 20 times higher in T lymphocytes than B lymphocytes. ${ }^{21}$ It is an enzymatic marker of cell immune response to $\mathrm{M}$ tuberculosis. ${ }^{31,32}$ Studies compare the ADA activity in pericardial effusions of variable etiologies and show that, considering the cut-off of $40 \mathrm{U} / \mathrm{l}$ for the diagnosis of tuberculous pericarditis, the specificity ranges from $72 \%-89 \%$ and the sensitivity from $87 \%-89 \%$ with VPP of $95 \%$ and VPN of $72 \% .{ }^{20,21}$ When this cut-off is reduced to $30 \mathrm{U} / \mathrm{I}$ the specificity falls to $68 \%$, but the sensitivity remains high $94 \% .{ }^{30}$ Regarding ADA activity in pericardial effusion, false positive results can be observed in septic pericarditis. Therefore, excluding this diagnostic possibility, the specificity for this enzymatic dosage increases, enabling the beginning of specific therapeutic. ${ }^{20}$ The co-infection by HIV did not show significant difference in the ADA activity compared to HIV negative patients. Our sample showed ADA activity higher than $40 \mathrm{U} / \mathrm{l}$ in $33 \%$ of the cases. Thus the low ADA activity did not allow us to exclude the diagnostic possibility of tuberculous pericarditis.

The polymerase chain reaction for Mycobacterium tuberculosis was tested in the pericardial effusion of 48 patients, out of which 33 were diagnosed with tuberculous pericarditis and showed sensitivity of $30 \%$ and specificity of $100 \% .{ }^{6}$ Another study tested the reaction in 13 samples of pericardial fluid and 15 samples of pericardial tissue of patients with definite diagnosis of tuberculous pericarditis. The sensitivity detected was $15 \%$ for the pericardial effusion and $81 \%$ for the pericardial tissue. This technique is less sensitive than other methods. The high specificity of this reaction may be compromised by contaminants, producing falsepositive results. Therefore, this technique is still not appropriate for routine clinical practice. ${ }^{9}$

\section{CONCLUSION}

Due to the considerable incidence of tuberculous pericarditis in our environment, physicians must be attentive to the clinical signs and symptoms. They should also be familiar with the diagnostic resources to perform the diagnosis and offer early treatment, providing better prognosis for these patients.

Clinical features, presence of signs of cardiac tamponade on echocardiogram and the histology of the pericardial fragment were the main data to reach the diagnosis of tuberculous pericarditis. The diagnostic index proposed by Reuter proved to be very useful in our sample with $100 \%$ sensitivity for tuberculous pericarditis diagnosis.

\section{REFERENCES}

1. Wolinsky E. Diseases due to mycobacteria. In: Wyngaarden JB, Smith LH Jr, editors. Cecil textbook of medicine. 18th ed. Philadelphia: WB Saunders; 1988. p.166293.

2. World Heath Organization. Tuberculosis [Internet]. [Place unknown]: WO; 2011 [cited 2011 Jan 28]. Available from: http://www.who.int/topics/tuberculosis/en/

3. Secretaria de Estado da Saúde (SP), Centro de Vigilância Epidemiológica Prof. Alexandre Vranjac. Di- visão de Controle da Tuberculose. Tuberculose em São Paulo [Internet]. São Paulo: CVE; [cited 2011 Jan 28]. Available from: http://www.cve.saude.sp.gov.br/htm/ cve_tb.html. Portuguese.

4. Scheepers GWH. Tuberculous Pericarditis. Am J Cardiol. 1962;9:248-76.

5. Imazio M, Spodick DH, Brucato A, Trincheiro R, Marckel $G$, Adler Y. Diagnostic issues in the clinical manegement of Pericarditis. Int J Clin Pract. 2010;64,(10):1384-92. 
6. Reuter H, Burgess L, Vuuren W van, Doubell A. Diagnosing Tuberculous Pericarditis. Q J M. 2006;99(12):827-39.

7. Sagrista-Sauleda J, Permanyer-Miranda G, Soler-Soler J. Tuberculous Pericarditis: ten year experience with a prospective protocol for diagnosis and treatment. J Am Coll Cardiol. 1988;11(4):724-8.

8. Mayoshi MB, Burgess LJ, Doubell AF. Tuberculous Pericarditis. Circulation. 2005;112(23):3608-16.

9. Desai HN. Tuberculous Pericarditis: a review of 100 cases. S Afr Med J. 1979;55(22):877-80.

10. Yang CC, Lee MH, Liu JW, Leu HS. Diagnosis of Tuberculous Pericarditis and treatment without corticosteroids in a tertiary teacher Hospital in Taiwan: a 14 years experience. J Microbiol Immunol Infect. 2005;38(1):47-52

11. Spodick DH. Tuberculous Pericarditis. Arch Intern Med. 1956;98(6):737-49.

12. Tirilomis T, Unverdorben S, Von der Emde J. Pericardectomy for chronic constrictive Pericarditis: risks and outcome. Eur J Cardiothoracic Surg. 1994;8(9):487-92.

13. Maher D, Harries AD. Tuberculous pericardial effusion: a prospective clinical study in a low-resource setting - Blantyre, Malawi. Int J Tuberc Lung Dis. 1997;1(4):358-64.

14. Hageman JH, D Esopo N, Glenn WWL. Tuberculosis of the pericardium: a long term analysis of forty-four proved cases. N Eng J Med. 1964;270:327-32.

15. Arroyo M, Soberman JE. Adenosine Deaminase in the diagnosis of tuberculous pericardial effusion. Am J Med Sci. 2008;335(3):227-9.

16. Yetkin U, Ilhan G, Calli AO, Yesil M, Gurbuz A. Severe calcific chronic constructive Tuberculous Pericarditis. Tex Heart Inst J. 2008;35(2):224-5.

17. Smedema JP, Katjitae I, Reuter H, ET al. Twelve - lead electrocardiography in Tuberculous Pericarditis. Cardiovasc J S Afr. 2001;12(1):31-4.

18. Light RW. Pleural effusions. Med Clin North Am. 1977;61(6):1339-52.

19. Light RW, Mac Gregor MI, Luchsinger PC, Ball WC. Pleural effusion: the diagnostic separation of transudates and exudates. Ann Intern Med. 1972;77(4):507-13.
20. Tuon FF, Silva VL da, Almeida GD de, Antonangelo L, Ho YL. The usefulness of adenosine Deaminase in the diagnosis of Tuberculous Pericarditis. Rev Inst Med Trop São Paulo. 2007,49(3):165-70.

21. Reuter H, Burgess LJ, Schneider J,Van Vuuren W, Doubell AF. The role of histopathology in establishing the diagnosis of tuberculous pericardial effusion in the presence of HIV. Histopathology. 2006;48(3):295-302.

22. Fowler NO. Tuberculous Pericarditis. JAMA. $1991 ; 266(1): 99-103$.

23. Strang G, Latouf $S$, Commerford P, et al. Bedside culture to confirm Tuberculous Pericarditis. Lancet.1991;338(878 2/8783):1600-1.

24. Strang JIG, Kakaza HHS, Gibson DG, et al Controlled clinical trial of complete open surgical drainage and of prednisolone in treatment of Tuberculous pericardial effusion in Transkei. Lancet. 1988;2(8614):759-64.

25. Barr JF. The use of pericardial biopsy in establishing etiologic diagnosis in acute Pericarditis. Arch Intern Med. 1955;96(5):693-6.

26. Vianna CB, Barreto ACP, Mady C, ET al. Pericardite tuberculosa: dificuldades diagnósticas e valor da biópsia pericárdica. Arq Bras Cardiol. 1986;47(1):27-30.

27. Rooney JJ, Crocco JA, Lyons HA. Tuberculous Pericarditis. Ann Intern Med. 1970;72;(1)73-81.

28. Quale JM, Lipschik GY, Heurich AE. Management of Tuberculous Pericarditis. Ann Thorac Surg.1987;43(6):653-5.

29. Guidugli RB, Hamrick PA, Rezende NF. Tuberculous Pericarditis in acquired immune deficiency syndrome patients. J Pneumol. 2003;29(2):98-100.

30. Burges LJ, Reuter H, Carstens M, Taljaard F, Doubell A. The use of adenosine Deaminase and interferon- $y$ as diagnostic tools for Tuberculous Pericarditis. Chest. 2002;122(3):900-5.

31. Blake J, Berman P. The use of adenosine Deaminase assays in the diagnosis of tuberculosis. S Afr Med J.1982;62(1):19-21.

32. Konsuoglu B, Goldeli O, Kulan K, Konsuoglu SS. The diagnostic and prognostic value of adenosine Deaminase in Tuberculous Pericarditis. Europ Heart J. 1995;16(8):1126-30.

\section{Conflict of interest: None}

Submitted on: 16th January 2011

Accept on: 15th March 2011

Correspondence: Divisão de Clínica Médica

Av. Prof. Lineu Prestes 2565 - Cidade Universitária - USP - São Paulo - SP - Brazil

CEP 05508-900 - Tel.: 55.011. 3091-9200

E-mail: ffcampos@usp.br 